\title{
The Effects of Gender and Multiple Oral Dosing on the Pharmacokinetics and Bioavailability of Morroniside in Beagle Dogs: A Pilot Study
}

\author{
S. XIONG ${ }^{1 *}$, J. LI ${ }^{1}$, Y. MU, X. ZHU ${ }^{1,2}$, X. WANG ${ }^{1}$ AND Z. ZHANG ${ }^{1 *}$
}

Institute of Materia Medica, Shandong Academy of Medical Sciences, Jinan-250062, ${ }^{1}$ Key Laboratory of Drug Metabolism and Pharmacokinetics, Beijing Institute of Pharmacology and Toxicology, Beijing-100850, 2Department of Pharmacy, Guangzhou Hui-Ai Hospital, Guangzhou-510370, China

Xiong, et al.: Gender and Multiple Oral Dosing Effects on Pharmacokinetics and Bioavailability of Morroniside

\begin{abstract}
Morroniside is a well-known iridoid plant glycoside. Attempts were made to study the effects of gender and multiple oral dosing on the pharmacokinetics and bioavailability of morroniside in beagle dogs. The concentration of morroniside in plasma was determined using a liquid chromatography tandem-mass spectrometry method. Main pharmacokinetic parameters were estimated by DAS 2.0 Pharmacokinetic software. Statistical analysis was performed using student t-test with P-values less than 0.05 as the level of significance. No significant gender difference was observed in the pharmacokinetic behaviour of morroniside and no differences were observed in the pharmacokinetic parameters following single and multiple administrations of morroniside. The absolute bioavailability after the oral administration of morroniside in beagle dogs was found to be $7.47 \pm 0.65 \%$.
\end{abstract}

Key words: Morroniside, LC-MS/MS, pharmacokinetics, bioavailability, beagle dogs

Morroniside, an iridoid glycoside (fig. 1), is the main active ingredient from Cornus officinalis Sieb. et Zucc. and Sambucus williamsii Hance, both of which are rich sources of iridoid glycosides and have been used as traditional Chinese medicinal herbs for centuries. Various pharmacologic studies have indicated that morroniside has therapeutic effects on diabetic angiopathies, renal damage, lipid metabolism and inflammation and bone resorption ${ }^{[1-4]}$. In recent studies, morroniside showed the protective actions against the cytotoxicity produced by exposure to $\mathrm{H}_{2} \mathrm{O}_{2}$ in human SH-SY5Y cells ${ }^{[5,6]}$. In addition, morroniside could protect ischemia/reperfusion-induced brain injury by decreased the caspase- 3 activity, reduced the infarction volume and minimized oxidative stress ${ }^{[7]}$.

There has been active interest in recent years in developing and optimizing analytical methods for determination of morroniside in rat plasma samples using high-performance liquid chromatography (HPLC) with ultraviolet (UV) detection ${ }^{[8]}$. However, only few reports have addressed the pharmacokinetics of morroniside in beagle $\operatorname{dogs}^{[9]}$. In our previous work, the analysis of morroniside in beagle dog plasma using liquid chromatography tandem-mass spectrometry (LC-MS/MS) has been systematically validated ${ }^{[9]}$.

However, up to now, there were no reports on the pharmacokinetic properties including gender-related pharmacokinetic differences, pharmacokinetic behaviour after multiple dosing, and oral bioavailability of morroniside in beagle dogs. Therefore, it was necessary for an intensive investigation on pharmacokinetics of morroniside. In the present study, the pharmacokinetics of morroniside after oral administration were systematically investigated using a well-validated sensitive LC-MS/MS method. The key pharmacokinetic issues mentioned above would be well addressed in support of the development of morroniside as a candidate drug.

This is an open access article distributed under the terms of the Creative Commons Attribution-NonCommercial-ShareAlike 3.0 License, which allows others to remix, tweak, and build upon the work non-commercially, as long as the author is credited and the new creations are licensed under the identical terms

Accepted 25 March 2017

Revised 09 November 2016

Received 16 October 2015

Indian J Pharm Sci 2017;79(2):303-306 


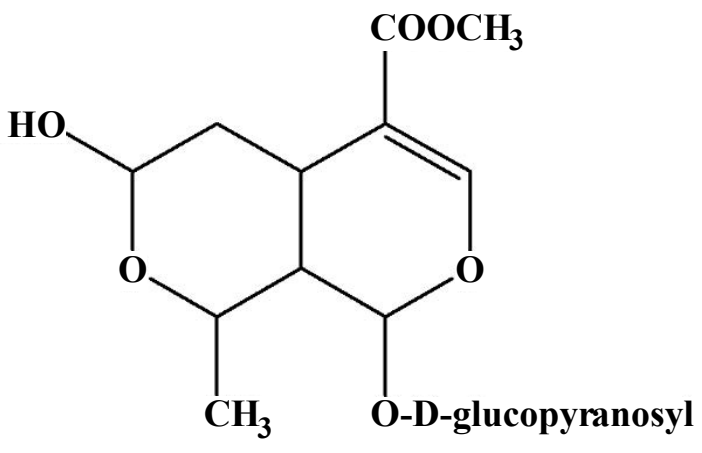

Fig. 1: Chemical structure of morroniside

Morroniside was extracted and purified from sarcocarp of C. officinalis, in the Department of Pharmacology, Xuanwu Hospital of Capital Medical University and its purity was over $98.5 \%$ by HPLC analysis. Paeoniflorin, used as the internal standard (IS), was purchased from the National Institute for the Control of Pharmaceutical and Biological Products (Beijing, China). HPLC-grade methanol and acetonitrile were obtained from Fisher Scientific (Fair Lawn, NJ, USA). Sodium formate was obtained from Beijing Chemical Reagent Company. All other chemicals were of analytic grade or better.

All analysis was performed with an API 5000 triplequadrupole mass spectrometer (Applied Biosystems/ MDS SCIEX, Foster City, CA, USA) coupled with electrospray ionization (ESI) source, which was operated in the positive ion mode. The liquid chromatography system consisted of a Shimadzu DGU-20A $\mathrm{A}_{5}$ degasser, two Shimadzu LC-20AD pumps, and a Shimadzu SIL-20AHT auto sampler (Shimadzu Scientific Instruments, Columbia, MD, USA).

An Inertsil ODS-SP column $(2.1 \times 50 \mathrm{~mm}, 5 \mu \mathrm{m})$ was adopted, with a mobile phase consisted of $1 \mathrm{mM}$ sodium formate (solvent A) and acetonitrile (solvent B) at a flow rate of $0.4 \mathrm{ml} / \mathrm{min}$ and the total run time was $6 \mathrm{~min}$ at room temperature. The gradient elution program was as follows: $5 \% \mathrm{~B}$ going to $20 \% \mathrm{~B}$ from 0-0.5 min, $20 \% \mathrm{~B}$ going to $70 \% \mathrm{~B}$ from $0.5-1.0 \mathrm{~min}$, $70 \% \mathrm{~B}$ going to $95 \% \mathrm{~B}$ from $1.0-3.0 \mathrm{~min}, 95 \% \mathrm{~B}$ going to $5 \% \mathrm{~B}$ from $3.0-3.2 \mathrm{~min}$ and finally $5 \% \mathrm{~B}$ from $3.2-$ $6.0 \mathrm{~min}$.

The mass spectrometer was operated in the multiple reactions monitoring (MRM) scan type. The transitions from molecular ion to dominant product ion $\mathrm{m} / \mathrm{z}$ $429 \rightarrow 267[\mathrm{M}+\mathrm{Na}]^{+}$and $m / z 503 \rightarrow 341[\mathrm{M}+\mathrm{Na}]^{+}$were monitored for morroniside and IS, respectively. The ESI source was operated with ion spray voltage at $5000 \mathrm{~V}$ and heater temperature at $550^{\circ}$. The optimized working parameters for mass detection were as follows:
CUR 25 1/h, CAD 12 1/h, Gas 145 1/h, Gas2 45 l/h, DP $180 \mathrm{~V}$, EP $10 \mathrm{~V}$, CE $35 \mathrm{~V}$ and CXP $15 \mathrm{~V}$. Analyst 1.5.2 software (AB SCIEX, Foster City, CA, USA) was used for system control and data processing ${ }^{[9]}$.

Twelve beagle dogs (nine males and three females), weighing $10.0 \pm 2.0 \mathrm{~kg}$ were purchased from Beijing Marshall Biological Technology Co., Ltd. (Beijing, China, Certificate number. SCXK, Beijing, 2011-0003). Room environment was maintained continuously at a temperature of $20-25^{\circ}$, relative humidity of $40-70 \%$, 10-15 air changes per hour and a $12 \mathrm{~h}$ fluorescent light/12 h dark cycle. All dogs were fed with standard dog diet and had free access to water. The animal experiments were conducted at the Beijing Center for Drug Safety Evaluation followed the protocol of the Institutional Animal Care and Use Committee of the Centre, which was in compliance with the guidelines of the Association for Assessment and Accreditation of Laboratory Animal Care International.

Dogs were fasted for at least $12 \mathrm{~h}$ with free access to water prior to the pharmacokinetic studies ${ }^{[10-13]}$. Three male and three female dogs were used to determine gender-related difference of morroniside. The dogs were administered with morroniside at a dose of 15 $\mathrm{mg} / \mathrm{kg}$ intragastrically (i.g.). Blood samples, about 1 $\mathrm{ml}$ each collected through hind leg vein, were placed in heparinized tubes pre-dose and at 5, 15, 30, 45, 60, 120, 240, 360, 480, and 720 min post-dose.

The dogs in multiple ( 7 times, $\mathrm{q} 8 \mathrm{~h}$ ) administrations group ( $\mathrm{n}=3)$ were also administered a dose of $15 \mathrm{mg} /$ $\mathrm{kg}$. Blood samples of multiple doses were immediately collected after the first and seventh oral administration at the same time point as single dose study.

In the single intravenous administration group, morroniside was administered intravenously (i.v.) through the fore leg vein at a dose of $5 \mathrm{mg} / \mathrm{kg}$. Blood samples were collected from the hind leg vein into heparinized tubes at $0,2,5,15,30,45,60,120,240$, 360,480 , and 720 min after administration.

All plasma samples were harvested by centrifugation at $3000 \mathrm{rpm} / \mathrm{min}$ for $15 \mathrm{~min}$ at $4^{\circ}$ and stored below $-20^{\circ}$ until LC-MS/MS analysis. Samples were prepared by using the standard protein precipitation method consisted of adding $50 \mu \mathrm{l}$ of plasma to a centrifuge vial, followed by $50 \mu \mathrm{l}$ of purified water and $350 \mu \mathrm{l}$ of methanol containing $100 \mathrm{ng} / \mathrm{ml}$ paeoniflorin. The tubes were vortex-mixed for $1 \mathrm{~min}$ and centrifuged at 14000 $\mathrm{rpm} / \mathrm{min}$ for $10 \mathrm{~min}$ and $10 \mu \mathrm{l}$ of the supernatant was analysed ${ }^{[9]}$. 
Absolute bioavailability (F\%) of morroniside was calculated using the following Eqn., $\mathrm{F} \%=\left(A U C_{(0-\infty,}\right.$ i.g.) $\left.\times D_{i . v .} / A U C_{(0-\infty, i . v .)} \times D_{\text {i.g. }}\right) \times 100$, (1), where $A U C_{(0-\infty \text {, }}$ i.g.) and $A U C_{(0-\infty, i . v .)}$ represent the area under the concentration-time curve from zero to infinity after morroniside oral and i.v. administration, respectively, whereas $D_{i . g .}$ and $D_{i . v .}$ represent the dose of morroniside oral and i.v. administration, respectively.

Data were given as mean \pm standard deviation (SD). Statistical analyses were performed using the SPSS 18.0 software package (SPSS Inc., Chicago, IL, USA) and pharmacokinetic parameters for morroniside were calculated by non-compartmental analysis using the Drug and Statistic (DAS) 2.0 pharmacokinetic software (Mathematical Pharmacology Professional Committee of China, Shanghai, China). Statistical analysis of the data was performed using student $t$-test. A different of $\mathrm{P}<0.05$ was considered as statistically significant.

The mean plasma concentration-time profiles of morroniside in beagle dogs were shown in fig. 2 .

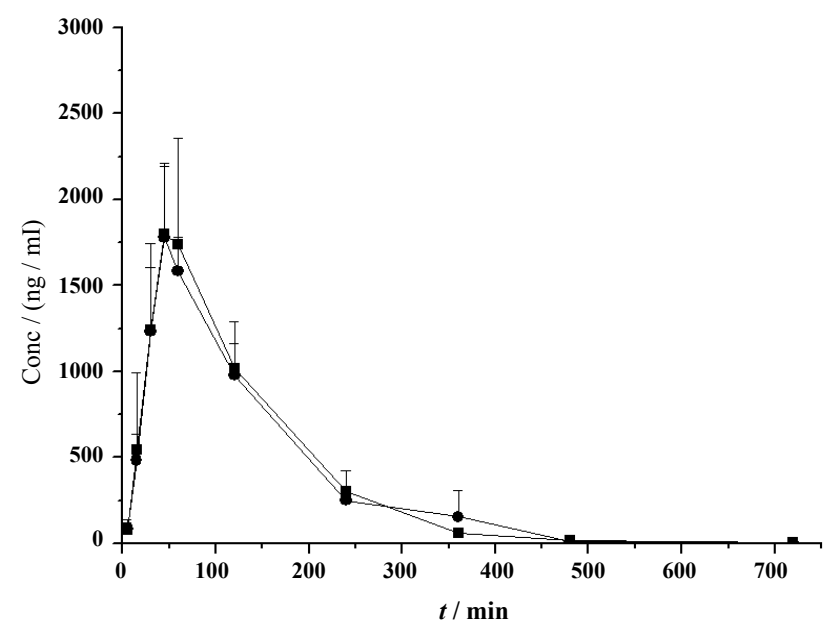

Fig. 2: Plasma concentration-time profiles of morroniside gender influence

Plasma concentration-time profiles of morroniside following

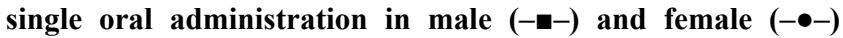
beagle dogs
Morroniside was detected in beagle dog plasma 5 min after oral administration. There were no genderrelated differences in the pharmacokinetic profiles of morroniside. The pharmacokinetic parameters of morroniside were summarized in Table 1 . No significant differences in $\mathrm{T}_{\max }(0.75 \pm 0.25 \mathrm{~h}$ vs. $0.83 \pm 0.14 \mathrm{~h}), \mathrm{C}_{\max }$ $(1886.67 \pm 533.14 \mathrm{ng} / \mathrm{ml}$ vs. $1800.00 \pm 391.28 \mathrm{ng} / \mathrm{ml})$, $A U C_{(0-\infty)}(4272.47 \pm 838.34 \mathrm{ng} \cdot \mathrm{h} / \mathrm{ml}$ vs. $4146.29 \pm 408.63$ $\mathrm{ng} \cdot \mathrm{h} / \mathrm{ml}), t_{1 / 2 z}(1.13 \pm 0.14 \mathrm{~h}$ vs. $0.87 \pm 0.04 \mathrm{~h}), C L_{z}$ $(3.60 \pm 0.671 / \mathrm{h} / \mathrm{kg}$ vs. $3.64 \pm 0.341 / \mathrm{h} / \mathrm{kg})$ and $M R T$ $(1.93 \pm 0.31 \mathrm{~h}$ vs. $2.04 \pm 0.31 \mathrm{~h})$ were observed between the male and female beagle dogs.

The mean plasma concentration-time profiles after single and multiple oral administrations of morroniside in beagle dogs were shown in fig. 3 and the derived pharmacokinetic parameters were presented in Table 1 . Morroniside concentrations reached a maximum of $1940.00 \pm 289.31 \mathrm{ng} / \mathrm{ml}$, with the $A U C_{(0-\infty)}, t_{1 / 2 z}, C L_{z}$ and $M R T$ equal to $4128.92 \pm 1259.65 \mathrm{ng} \cdot \mathrm{h} / \mathrm{ml}, 0.97 \pm 0.05 \mathrm{~h}$, $3.91 \pm 1.36 \mathrm{l} / \mathrm{h} / \mathrm{kg}$ and $1.60 \pm 0.21 \mathrm{~h}$ following multiple oral administrations. The absorption of morroniside for multiple administrations was still very fast $\left(\mathrm{T}_{\max }\right.$, $0.57 \pm 0.13 \mathrm{~h}$ ). No significant deviation was observed in the difference of all the pharmacokinetic parameters following the single and multiple doses of morroniside in dogs.

The mean plasma concentration-time profile after i.v. administration of morroniside to beagle dogs was shown in fig. 4 with the corresponding pharmacokinetic parameters given in Table 1. After dosage correction, the oral bioavailability of morroniside was calculated by comparing the corresponding values of $A U C_{(0-\infty)}$. The results showed that oral administration of morroniside led only to an absolute bioavailability of $(7.47 \pm 0.65 \%)$.

In summary, this was the first study to investigate the pharmacokinetic characteristics of morroniside including gender-dependent pharmacokinetic differences,

\section{TABLE 1: PHARMACOKINETIC PARAMETERS OF MORRONISIDE IN BEAGLE DOGS}

\begin{tabular}{ccccc}
\hline \multirow{2}{*}{ Parameter/ data unit } & \multicolumn{4}{c}{ Parameter value/mg $\mathbf{k g}^{-1}$} \\
\cline { 2 - 5 } & $\mathbf{1 5}$ (male, i.g., single) & $\begin{array}{c}\mathbf{1 5} \text { (female, i.g., } \\
\text { single) }\end{array}$ & $\begin{array}{c}\mathbf{1 5} \text { (male, i.g., } \\
\text { multiple) }\end{array}$ & $\mathbf{5}$ (female, i.v., single) \\
\hline $\mathrm{T}_{\max } / \mathrm{h}$ & $0.75 \pm 0.25$ & $0.83 \pm 0.14$ & $0.57 \pm 0.13$ & $0.03 \pm 0.00$ \\
$C_{\max } / \mathrm{ng} \cdot \mathrm{ml}^{-1}$ & $1886.67 \pm 533.14$ & $1800.00 \pm 391.28$ & $1940.00 \pm 289.31$ & $25900.00 \pm 1967.23$ \\
$A U C_{(0-t)} / \mathrm{ng} \cdot \mathrm{h} \cdot \mathrm{ml}^{-1}$ & $4267.89 \pm 834.60$ & $4133.04 \pm 406.93$ & $4128.03 \pm 1259.44$ & $18933.22 \pm 2021.30$ \\
$A U C_{(0-\infty)} / \mathrm{ng} \cdot \mathrm{h} \cdot \mathrm{ml}^{-1}$ & $4272.47 \pm 838.34$ & $4146.29 \pm 408.63$ & $4128.92 \pm 1259.65$ & $18945.09 \pm 2007.92$ \\
$t_{1 / 2 z} / \mathrm{h}$ & $1.13 \pm 0.14$ & $0.87 \pm 0.04$ & $0.97 \pm 0.05$ & $0.92 \pm 0.06$ \\
$C L_{z} / l \cdot \mathrm{h}^{-1} \cdot \mathrm{kg}^{-1}$ & $3.60 \pm 0.67$ & $3.64 \pm 0.34$ & $3.91 \pm 1.36$ & $0.27 \pm 0.03$ \\
$M R T / \mathrm{h}$ & $1.93 \pm 0.31$ & $2.04 \pm 0.31$ & $1.60 \pm 0.21$ & $1.02 \pm 0.05$ \\
$\mathrm{~F} / \%$ & $7.47 \pm 0.65$ & & & \\
\hline
\end{tabular}

i.g. (intragastric); i.v. (intravenous). Data are expressed as mean \pm standard deviation $(n=3)$ 


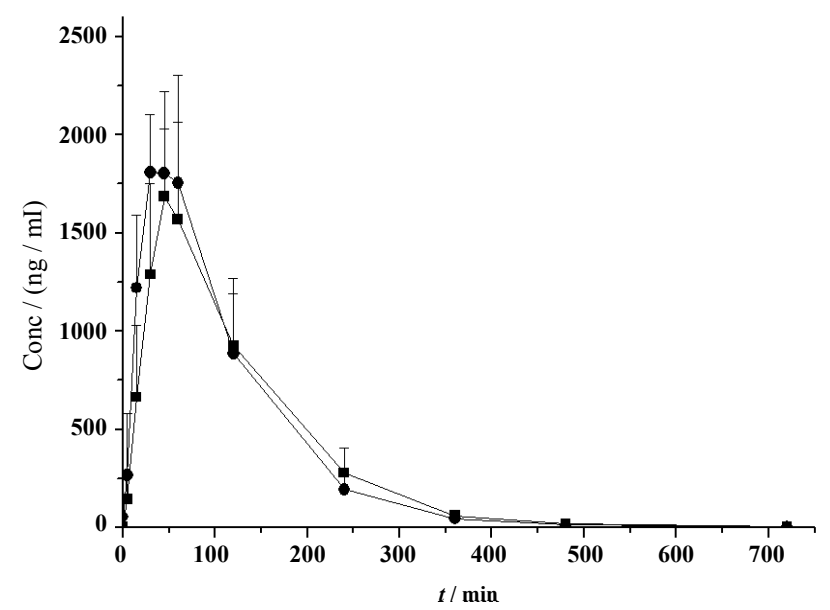

Fig. 3: Plasma concentration-time profiles of morroniside single and multiple dosing

Plasma concentration-time profiles of morroniside following single (--) and multiple $(-\bullet-)$ oral administrations in male beagle dogs

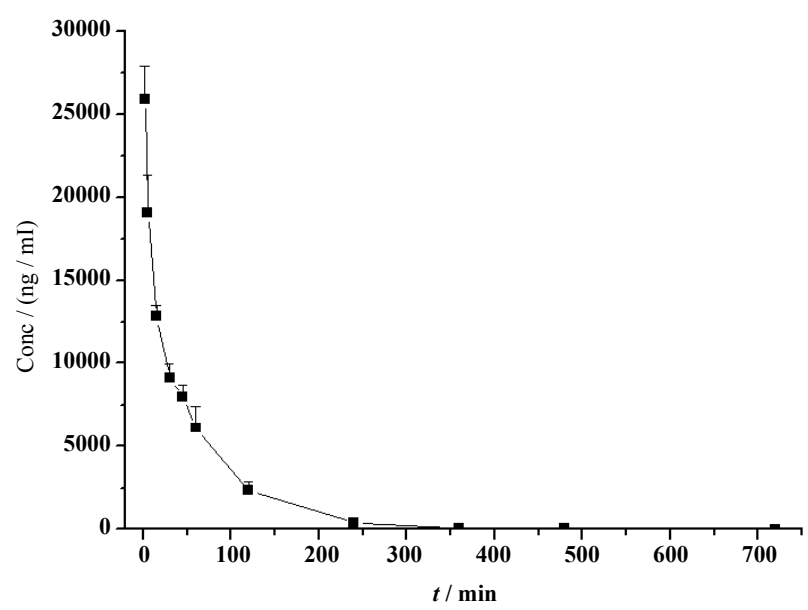

Fig. 4: Plasma concentration-time profile of morroniside Plasma concentration-time profile of morroniside following single intravenous injection (--) in male beagle dogs

pharmacokinetic behaviours after multiple oral administrations, and oral bioavailability in beagle dogs.

Our results provided valuable pharmacokinetic information for rational use of morroniside. The pharmacokinetic parameters of morroniside were important to elucidate its pharmacological properties and further investigations were required to reveal the underlying mechanisms for absorption and metabolism of morroniside in vivo.

\section{Conflict of interests:}

Authors report no conflict of interests.

\section{Financial support and scholarship:}

This work has been financially supported by the
Innovation Project of Shandong Academy of Medical Sciences and National Major Scientific and Technical Special Projects for Innovative Drug of China (No. 2012ZX09301003- 001-007).

\section{REFERENCES}

1. Xu HQ, Hao HP, Zhang X, Pan Y. Morroniside protects cultured human umbilical vein endothelial cells from damage by high ambient glucose. Acta Pharmacol Sin 2004;25:412.

2. Yokozawa T, Yamabe N, Kim HY, Kang KS, Hur JM, Park $\mathrm{CH}$, et al. Protective effects of morroniside isolated from Corni fructus against renal damage in streptozotocin-induced diabetic rats. Biol Pharm Bull 2008;31:1422-8.

3. Park CH, Yamabe N, Noh JS, Kang KS, Tanaka T, Yokozawa $\mathrm{T}$. The beneficial effects of morroniside on the inflammatory response and lipid metabolism in the liver of $\mathrm{db} / \mathrm{db}$ mice. Biol Pharm Bull 2009;32:1734-40.

4. Li M, Wang W, Wang P, Yang K, Sun H, Wang X. The pharmacological effects of morroniside and loganin isolated from Liuweidihuang Wan, on MC3T3-E1 cells. Molecules 2010;15:7403-14.

5. Wang W, Huang W, Li L, Ai H, Sun F, Liu C, et al. Morroniside prevents peroxide-induced apoptosis by induction of endogenous glutathione in human neuroblastoma cells. Cell Mol Neurobiol 2008;28:293-305.

6. Wang W, Xu J, Li L, Wang P, Ji X, Ai H, et al. Neuroprotective effect of morroniside on focal cerebral ischemia in rats. Brain Res Bull 2010;83:196-201.

7. Wang W, Sun F, An Y, Ai H, Zhang L, Huang W, et al. Morroniside protects human neuroblastoma SH-SY5Y cells against hydrogen peroxide-induced cytotoxicity. Eur J Pharmacol 2009;613:19-23.

8. Li X, Wang Q, Zhang X, Sheng X, Zhou Y, Li M, et al. HPLC study of pharmacokinetics and tissue distribution of morroniside in rats. J Pharm Biomed Anal 2007;45:349-55.

9. Xiong S, Li J, Zhu X, Wang X, Lü G, Zhang Z. Determination of morroniside concentration in beagle plasma and its pharmacokinetics by high performance liquid chromatographytandem mass spectrometry. Se Pu 2014;32:290-3.

10. Shanmugasundaram S, Manjunatha N, Vijayan R, Khatwal RB, Samanta MK. Determination and estimation of pharmacokinetic profile of caffeine in form of extract of green tea leaves and its analogy with synthetic form. Indian J Pharm Sci 2011;73:649-55.

11. Xu H, Zhang T, Yang H, Xiao X, Bian Y, Si D, et al. Preparation of evodiamine solid dispersions and its pharmacokinetics. Indian J Pharm Sci 2011;73:276-81.

12. Katayama M, Kawakami Y, Katayama R, Shimamura S, Okamura Y, Uzuka Y. Preliminary study of effects of multiple oral dosing of clarithromycin on the pharmacokinetics of cyclosporine in dogs. J Vet Med Sci 2014;76:431-3.

13. Mahmoud MA, Ebid AH, Shouman SA, Ebid EN. Pharmacokinetics of vancomycin in oncology Egyptian paediatrics: a dosage adjustment trial. Indian J Pharm Sci 2014;76:82-6. 